\title{
Therapeutic evaluation of arterio-portal fistula-related gastroesophageal variceal bleeding
}

\author{
Xiaoquan Huang ${ }^{1}$, Wen Zhang ${ }^{2}$, Shiyao Chen ${ }^{1}$, Chengfeng Liu' ${ }^{1}$, Ruofan Sheng ${ }^{3}$, \\ Feng $\mathbf{L i}^{1}$, Jian Wang ${ }^{1}$, Jianjun Luo $^{2}$ and Pengju $\mathbf{X u}^{3}$ \\ ${ }^{1}$ Department of Gastroenterology, Zhongshan Hospital, Fudan University, Shanghai, China \\ 2 Department of Interventional Radiology, Zhongshan Hospital, Fudan University, Shanghai, China \\ ${ }^{3}$ Department of Radiology, Zhongshan Hospital, Fudan University, Shanghai, China \\ Correspondence to: Shiyao Chen, email: Chen.shiyao@zs-hospital.sh.cn \\ Keywords: combination treatment; portal hypertension; intrahepatic arterio-portal fistula; upper gastrointestinal bleeding \\ Received: January 31, $2017 \quad$ Accepted: March 14, 2017 \\ Published: March 25, 2017
}

Copyright: Huang et al. This is an open-access article distributed under the terms of the Creative Commons Attribution License 3.0 (CC BY 3.0), which permits unrestricted use, distribution, and reproduction in any medium, provided the original author and source are credited.

\section{ABSTRACT}

Background \& Aims: Intrahepatic arterio-portal fistula is an uncommon etiology of portal hypertension, which presents diagnostic and therapeutic challenges. This study aimed to assess the efficacy and outcomes of gastroesophageal variceal bleeding caused by arterio-portal fistula using different therapeutic approaches.

Methods: Medical records of $\mathbf{4 5 1}$ consecutive patients with arterio-portal fistula were reviewed from January 1, 2009, to July 15, 2016, and patients suffered variceal bleeding were eligible for the study.

Results: Among 57 patients with arterio-portal fistula, hepatocellular carcinoma was existed in $61.4 \%$ patients. A combination of radiological intervention and endoscopic treatment was performed in $8(14.0 \%)$ patients; the remainder were treated using radiological intervention alone $(n=20,35.1 \%)$, endoscopic treatment alone $(n=18,31.6 \%)$, or without any intervention $(n=11,19.3 \%)$. No patient died in the combination group, while 20 patients in the single-treatment group and 6 in the untreated group died during follow-up. A significant difference in the survival rate was found between the combination group and the other two groups. Treatment selection between combination and untreated groups was the only parameter significantly associated with survival $(p=0.002)$.

Conclusions: For patients diagnosed with arterio-portal fistula, combination treatment is the most optimal strategy in managing variceal bleeding, especially in patient with severe type of fistula.

\section{INTRODUCTION}

Intrahepatic arterio-portal fistula (IAPF) is an uncommon etiology of portal hypertension, which presents diagnostic and therapeutic challenges. Because of the communication between the hepatic artery and the portal vein, intrasinusoidal portal hypertension may result in ascites, hepatic encephalopathy, and varices with lifethreatening gastrointestinal hemorrhage [1][2]. Fewer than $10 \%$ cases of IAPF are congenital, and most cases of IAPF are secondary to liver tumors, interventional hepatic procedures, cirrhosis, and so on [3]. The diagnosis of IAPF is usually made accidentally on radiographic evaluation of the portal circulation. However, these patients are easy to be misdiagnosed or miss diagnosed at the first presentation of upper gastrointestinal bleeding (UGIB). Limited data are available on the management of gastroesophageal variceal bleeding in these patients. At present, hepatic arteriography is the gold standard in diagnosing IAPF and may also play a therapeutic role [4]. A review of patients with IAPF reported that transcatheter arterial embolization (TAE), an interventional radiological procedure, is the predominant therapeutic choice because of its low invasiveness [5]. Nevertheless, some of the patients suffered refractory variceal bleeding after radiologic intervention and even underwent surgical 
ligation or resection of the hepatic lobe [1][2]. Endoscopic treatments including $N$-butyl-2-cyanoacrylate injection for gastric varices and endoscopic ligation or sclerotherapy for esophageal varices have never been reported as the therapeutic approach to UGIB in patients with IAPF.

Therefore, the aim of this retrospective study was to evaluate the efficacy and outcomes of gastroesophageal variceal bleeding caused by IAPF using different therapeutic approaches.

\section{PATIENTS AND METHODS}

\section{Study design}

This was a retrospective, tertiary hospital-based study. Between January 1, 2009, and July 15, 2016, 451 consecutive patients were diagnosed as $\operatorname{IAPF}(\mathrm{s})$ by contrast-enhanced computed tomography (CT) at Zhongshan Hospital, Fudan University, Shanghai, China. Clinical data were analyzed from prospectively collected data on the Portal Hypertension database, and from medical records. In all cases, the diagnosis of gastroesophageal varices was made by either upper digestive endoscopy or CT scanning. All patients who suffered variceal bleeding before or after the diagnosis of IAPF were eligible for the study.

The study protocol was conducted conforming to the guidelines of the Declaration of Helsinki, as reflected in the approval granted by the Institutional Human Research Committee.

Patients with IAPF were categorized according to the therapies they received. The patients in the singletreatment group were given either endoscopic treatment or radiologic intervention, whereas those in the combination treatment group received both endoscopic treatment and radiologic intervention in any sequence. The patients in the untreated group did not receive endoscopic or radiologic interventions.

The study initiation was set on the day when patients experienced the first variceal bleeding episode, and the end point was July 31, 2016. All the participants were followed up via phone calls, outpatients' clinic visits, and chart reviews. The primary outcome of the study was allcause death, and the secondary outcome was the incidence of rebleeding after treatment among patients receiving interventions.

\section{Definition}

Rebleeding was defined as new onset of hematemesis, coffee-ground vomitus, hematochezia, or melena according to the guideline [6]. Early diagnosis of IAPF was described as the diagnosis of IAPF before or 7 days within the first bleeding episode. A peripheral type of IAPF was defined as a fistula located under the right/ left branches of the portal vein, and a central type of IAPF was defined as a fistula located in the main portal vein. However, in a diffuse type of IAPF, fistulas involved both the main portal vein and branches in the liver. Based on the severity, IAPFs in the present study were classified into two groups, mild and severe, by the extent of the early enhancement of peripheral portal vein branches and the presence of wedge-shaped, transient peripheral areas of enhancement in the arterial phase, as previously described by Guzman and Choi et al [7][8]. Figure 1 demonstrates the $\mathrm{CT}$ angiography of patients with IAPF.

\section{Endoscopic treatment of varices}

The patients received endoscopic treatment of variceal bleeding for either management of acute variceal bleeding or secondary prophylaxis of rebleeding. Endoscopic procedures were performed using Olympus-240/260 Gastroscopy (Olympus, Tokyo, Japan) under propofol sedation. As for esophageal varices, endoscopic ligation or sclerotherapy was performed by two experienced endoscopic specialists. Endoscopic ligation was applied using the Six-Shooter Multi-Band Ligator (Cook Endoscopy, Inc., NC, USA) at $1 \mathrm{~cm}$ above the Z-line in a spirally ascending fashion, with no more than six bands used per session. As for sclerotherapy, an intravariceal or paravariceal injection of lauromacrogol (10-30 mL per session; Tianyu Pharmaceutical Co., Ltd., Xi'an, China) was started above the Z-line and proceeded until all the visible esophageal varices were treated. Usually, endoscopic ligation is the primary selection and sclerotherapy is performed during followup endoscopy in those small esophageal varices which ligation is impossible. As for gastric varices, the "sandwich technique" was used for an N-butyl-2cyanoacrylate (Compont Medical Adhesive, $0.5 \mathrm{ml} / \mathrm{tube}$; Beijing Compont Medical Devices Co., Ltd., Beijing, China) injection. After flushing the injection needles (NM-200L-0423; Olympus, Tokyo, Japan) with an isotonic sodium chloride solution, the gastric varices were injected with lauromacrogol $(4 \mathrm{ml})$, cyanoacrylate $(0.5-$ $5 \mathrm{ml})$, and lauromacrogol $(4 \mathrm{ml})$ again. The needle sheath was held at the puncture site for 3-4s to prevent leakage of cyanoacrylate. Follow-up endoscopy was performed at an interval of no less than 2 months and treatment was repeated until complete obliteration was achieved.

\section{Radiologic interventions of fistula}

Transarterial embolization (TAE) was applied as a radiologic intervention for treating IAPF. Under local anesthesia, the transfemoral approach was routine. Selective angiography of the celiac axis and hepatic artery was performed with a $5 \mathrm{~F} \mathrm{RH}$ catheter (Cook, 
Bloomington, IN, USA). The embolic agent, microspheres (Embosphere Microspheres, BioSphere Medical, diameters ranging from 500 to $1200 \mu \mathrm{m}$ ) and irregular PVA particles (500-1000 $\mu \mathrm{m}$, multiple vendors), multiple Tornado embolization microcoils (Cook, Bloomington, IN, USA), N-butyl-2-cyanoacrylate (Compont Medical Adhesive; Beijing Compont Medical Devices Co., Ltd., Beijing, China, $0.5 \mathrm{ml}$ ), or gelatin sponge particles (100$1400 \mu \mathrm{m})$ were selected by the velocity of the flow of fistula-feeding artery(s) and the size and location of the fistula. The end point was determined by the satisfactory occlusion and disappearance of the fistula on angiography. Multiple interventions may be necessary for large-size fistulas.

\section{Statistical analysis}

Continuous variables were expressed as the mean \pm standard error of mean or median (range) and compared using the Student's $t$ test. Categorical variables were described with constituent ratios and compared using the chi-square or Fisher's exact test. The Kaplan-Meier method with log-rank test was used to estimate the cumulative probability of rebleeding and death. The Cox regression model was used to evaluate potential risk factors for survival. The bootstrap technique was performed, and the sample was set at 500. Model selection was guided by the results of univariate analyses $(P<$
0.10 ), and the best statistical fit was identified through the stepwise algorithm. Calculations were made using SPSS 23.0 software (SPSS, IL, USA). All statistical analyses were two-sided tests $(P<0.05)$.

\section{RESULTS}

\section{Patient characteristics at first bleeding}

Among 451 consecutive patients diagnosed with IAPF by CT scanning, 228 (50.6\%) had esophageal and/ or gastric varices and $57(12.6 \%)$ suffered from cirrhotic variceal bleeding episode. The mean age at first bleeding was $54.3 \pm 1.7$ years (range, 22-79), and $70.2 \%$ of the patients were males. Hepatocellular carcinoma (HCC) was existed in $35(61.4 \%)$ patients. The median period retrospect to the first episode of variceal bleeding until the end point was 281 days (range, 2-3361). Most of the patients had mild $(n=20,35.1 \%)$ to severe $(n=26$, $45.6 \%)$ ascites at presentation of variceal bleeding. The study flowchart and outcomes are shown in Figure 2. The characteristics of these patients with IAPF and the laboratory values are listed in Table 1.

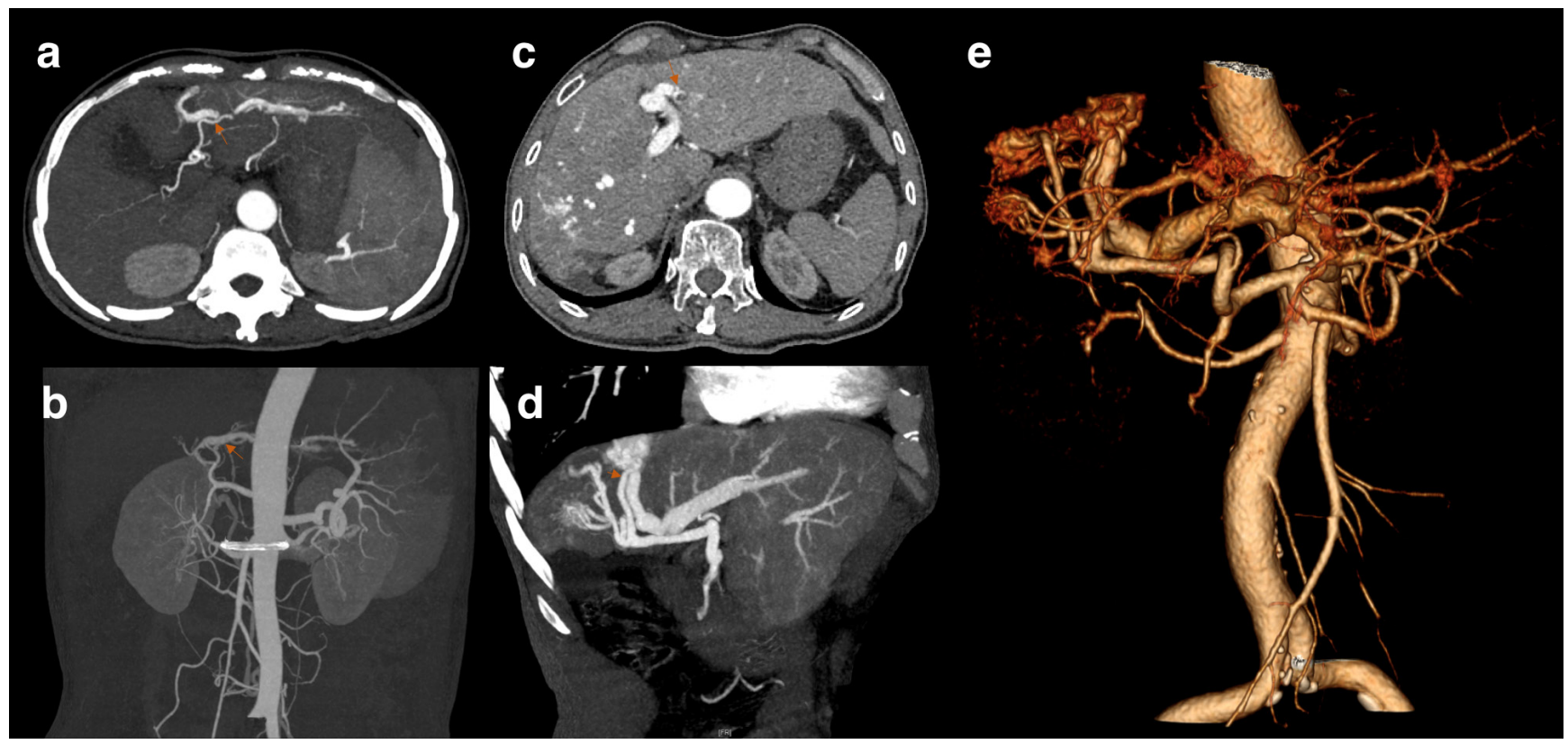

Figure 1: Figure a and $b$ are from the same patient and demonstrate the mild type of IAPF. a. The arterial phase of dynamic CT shows early enhancement of transient, peripheral, small hepatic vein branches. b. Maximal intensity projection (MIP) image of the portal trunk in the arterial phase shows the mild type of fistula. Figure c, d, and e are from the same patient and demonstrate the severe type of IAPF. c. Multiple fistulas developed in both central and peripheral areas. d. A large fistula was seen in the MIP image. e. Volume-rendered images show multiple fistulas in a severe type of IAPF. (Red arrows for concomitant hepatic arteries). 
Table 1: Patient demographics and baseline clinical and biochemical characteristics

\begin{tabular}{|c|c|}
\hline Variables & Total $(n=57)$ \\
\hline Age at first bleeding (year) & $54.3 \pm 1.7(2279)$ \\
\hline Age at diagnosis of IAPF & $55.0 \pm 1.7(2380)$ \\
\hline Early diagnosis of IAPF (yes/no) & $10(17.5 \%) / 47(82.5 \%)$ \\
\hline Sex (male/female) & $40(70.2 \%) / 17(29.8 \%)$ \\
\hline HBV (positive/negative) & $40(70.2 \%) / 17(29.8 \%)$ \\
\hline \multicolumn{2}{|l|}{ Etiology of IAPF } \\
\hline$-\mathrm{HCC}$ & $35(61.4 \%)$ \\
\hline -Cirrhosis & $18(31.6 \%)$ \\
\hline -AVM & $4(7.0 \%)$ \\
\hline Hemoglobin $(\mathrm{g} / \mathrm{dL})$ & $8.1 \pm 0.3(3.8-12.7)$ \\
\hline White blood cell count (109/L) & $4.4 \pm 0.3(0.86-16.3)$ \\
\hline Thrombocytes (103/L) & $94.5 \pm 7.8(29-275)$ \\
\hline Bilirubin $(\mu \mathrm{mol} / \mathrm{L})$ & $25.3 \pm 3.9(5.1-191.6)$ \\
\hline Albumin $(\mathrm{g} / \mathrm{L})$ & $30.7 \pm 0.7(19-43)$ \\
\hline Prothrombin time $(\mathrm{s})$ & $14.5 \pm 0.4(11.3-25.2)$ \\
\hline INR & $1.2 \pm 0.03(0.98-2.17)$ \\
\hline Creatinine $(\mu \mathrm{mol} / \mathrm{L})$ & $93.3 \pm 16.2(30-826)$ \\
\hline Child-Pugh Class (A/B/C) & $10(17.5 \%) / 35(61.4 \%) / 12(21.1 \%)$ \\
\hline MELD Score & $12.5 \pm 0.1(7-15)$ \\
\hline Splenectomy & $7(12.3 \%)$ \\
\hline Portal vein embolus & $35(61.4 \%)$ \\
\hline AFP (abnormal/normal) & $24(42.1 \%) / 33(57.9 \%)$ \\
\hline \multicolumn{2}{|l|}{ Ascites } \\
\hline -Absent & $11(19.3 \%)$ \\
\hline -Mild & $20(35.1 \%)$ \\
\hline -Severe & $26(45.6 \%)$ \\
\hline \multicolumn{2}{|l|}{ Hepatic encephalopathy } \\
\hline -Absent & $48(84.2 \%)$ \\
\hline -Mild & $8(14.0 \%)$ \\
\hline -Severe & $1(1.8 \%)$ \\
\hline Severity of IAPFs (mild/severe) & $25(43.9 \%) / 32(56.1 \%)$ \\
\hline \multicolumn{2}{|l|}{ Types of IAPFs } \\
\hline -Peripheral type & $26(45.6 \%)$ \\
\hline -Central type & $28(49.1 \%)$ \\
\hline -Diffuse type & $3(5.3 \%)$ \\
\hline
\end{tabular}

Continuous variables are expressed as mean \pm standard error of mean with range in parentheses.

The Child-Pugh score ranges from 5 to 15: class A (5-6 points), class B (7-9 points), and class C (10-15 points).

Abnormal AFP value is defined as $>20.0 \mathrm{ng} / \mathrm{mL}$.

AFP, Alpha fetal protein; AVM, arteriovenous malformation; HBV, hepatitis B virus; INR, international normalized ratio; MELD, Model for End-stage Liver Disease.

\section{Procedural characteristics}

IAPFs were diagnosed by contrast-enhanced CT scanning. Ten patients were diagnosed with IAPF before UGIB, while the other 47 patients were diagnosed with IAPF after variceal bleeding. Seven patients received splenectomy at the first presentation of UGIB before the diagnosis of IAPF. The interval from the first episode of bleeding until the diagnosis of IAPF as a possible etiological factor for portal hypertension was 21 days (range, -538 to 5438 ). A combination of radiological interventions and endoscopic treatment was performed in $14.0 \%(n=8)$ of patients, whereas the remainder were treated using radiological intervention alone $(n=20$, $35.1 \%)$, endoscopic treatment alone $(n=18,31.6 \%)$, or without any interventions $(n=11,19.3 \%)$. All the 28 patients received radiological interventions were confirmed IAFP by hepatic arteriography. 
Table 2: Univariate and multivariate analyses of overall survival

\begin{tabular}{|l|c|c|c|c|}
\hline \multicolumn{1}{|c|}{ Variable } & \multicolumn{3}{c|}{ OS } \\
\hline & \multicolumn{2}{|c|}{ Univariate analysis } & \multicolumn{2}{c|}{ Multivariate analysis } \\
\hline & $\mathbf{9 5 \%}$ confidence interval & $\boldsymbol{P}$ value & 95\% confidence interval & $\boldsymbol{P}$ value \\
\hline $\begin{array}{l}\text { Age at first bleeding } \\
(<55 \text { y vs. } \geq 55 \text { y) }\end{array}$ & $(-1.110) 0.441$ & 0.425 & & \\
\hline Sex (male vs. female) & $(-1.837) 0.164$ & 0.152 & & \\
\hline HCC & $0.641-2.701$ & 0.004 & $(-0.020)-3.192$ & 0.112 \\
\hline HBV & $0.109-1.985$ & 0.026 & & \\
\hline $\begin{array}{l}\text { Child-Pugh class } \\
\text { (A vs. B vs. C) }\end{array}$ & $0.462-1.695$ & 0.002 & $(-0.257)-1.827$ & 0.126 \\
\hline Severity of IAPFs & $(-0.047)-1.876$ & 0.040 & $(-0.459)-2.288$ & 0.257 \\
\hline Portal vein embolus & $(-0.126) 1.638$ & 0.090 & $(-0.737) 2.086$ & 0.393 \\
\hline Treatment selection & & & & \\
\hline Single vs. untreated & $(-1.875-0.967$ & 0.623 & $(-2.040)-1.203$ & 0.661 \\
\hline Combination vs. untreated & $(-15.947)-(-12.861)$ & 0.004 & $(-16.224)(-11.658)$ & 0.002 \\
\hline $\begin{array}{l}\text { Diagnosis of IAPFs before or 7 days within } \\
\text { bleeding }\end{array}$ & $(-2.370)-0.172$ & 0.014 & $(-3.043)-0.502$ & 0.236 \\
\hline
\end{tabular}

Eighteen patients received solely endoscopic treatments with an average of 1.7 (range, 1-5) procedures, and 20 patients received only TAE approaches with the mean of 1.5 (range, 1-4) procedures. Four patients first received endoscopic procedures before the diagnosis of IAPF, and three of them switched to TAE at the moment of finding the existence of IAPF. The other one chose not to receive TAE treatment until the second severe variceal bleeding and had the hepatic venous pressure gradient measurement of $22 \mathrm{mmHg}$. Four patients had their fistula embolized first; three of them suffered recurrent variceal bleeding and thus received the endoscopic treatment, and the other one received combination endoscopic treatment for secondary prophylaxis of variceal bleeding.

\section{Primary and secondary end points}

\section{Overall survival}

A total of $21(55.3 \%)$ patients in the single-treatment group [hypovolemic shock secondary to UGIB $(n=10)$; liver failure $(n=11)$ ] and $6(54.5 \%)$ patients [hypovolemic shock secondary to UGIB $(n=2)$, liver failure $(n=2)$, multi-organ dysfunction syndrome $(n=1)$, and unknown $(n=1)]$ in the untreated group died during the followup period. Four patients (three in the single-treatment group and one in the untreated group) underwent liver transplantation and were alive without further episodes of variceal bleeding. The cumulative survival rate at 1,2 , and 3 years was $100 \%, 100 \%$, and $100 \%$ in the combination treatment group, $63 \%, 45 \%$, and $39 \%$ in the single-

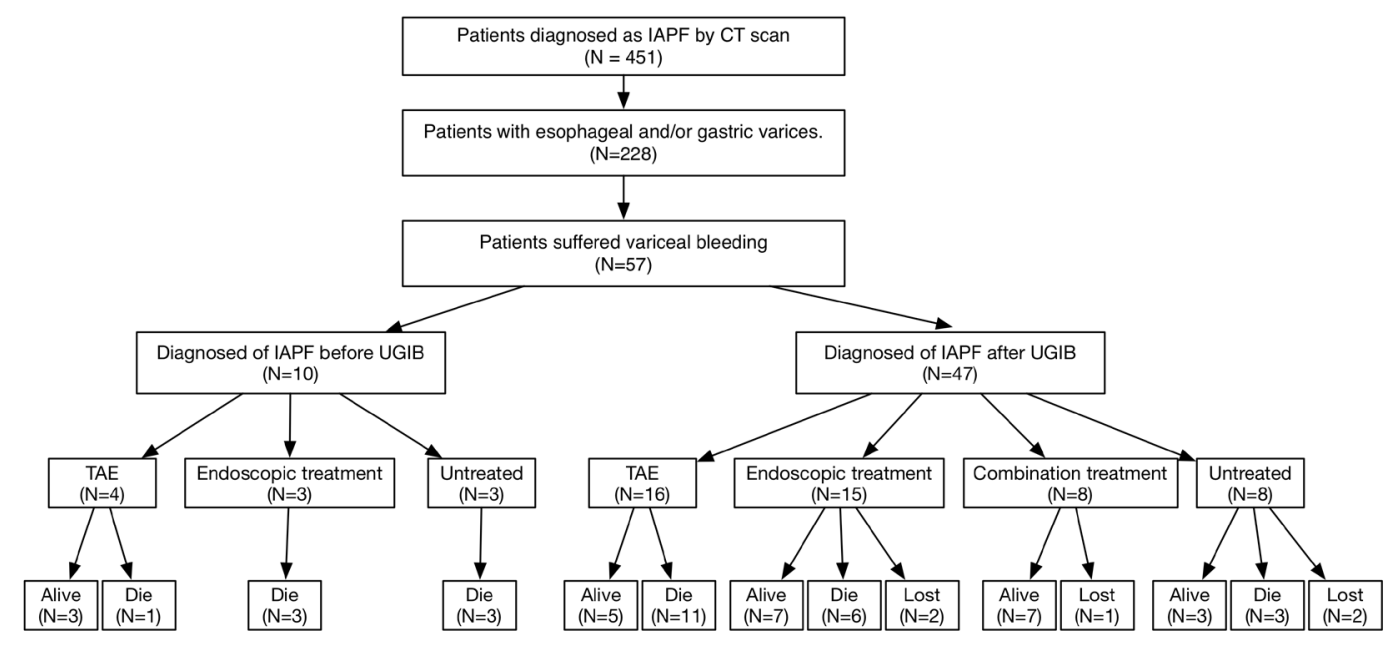

Figure 2: Study flowchart showing patients' inclusion and outcomes. 
Table 3: Rebleeding and outcomes in the group that first received endoscopic treatment and the group that first underwent TAE stratified by the severity of IAPF

\begin{tabular}{|l|c|c|c|c|}
\hline & \multicolumn{2}{|c|}{ Endoscopic treatment first $(\boldsymbol{n}=\mathbf{2 2})$} & \multicolumn{2}{|c|}{$\begin{array}{c}\text { TAE first } \\
(\boldsymbol{n}=\mathbf{2 4})\end{array}$} \\
\hline Severity of IAPF & Mild & Severe & Mild & Severe \\
\hline Rebleeding & $5(22.7 \%)$ & $7(31.8 \%)$ & $3(12.5 \%)$ & $11(45.8 \%)$ \\
\hline 7-day rebleeding & 0 & $2(9.1 \%)$ & 0 & 0 \\
\hline 3-month rebleeding & $2(9.1 \%)$ & $1(4.5 \%)$ & $3(12.5 \%)$ & $5(20.8 \%)$ \\
\hline 6-month rebleeding & $1(4.5 \%)$ & $2(9.1 \%)$ & 0 & $3(12.5 \%)$ \\
\hline 12-month rebleeding & $1(4.5 \%)$ & 0 & 0 & $2(8.3 \%)$ \\
\hline $\begin{array}{l}\text { Interval between first treatment and rebleeding } \\
\text { (days) }\end{array}$ & 138 & 137 & 253 & 115 \\
\hline Death & $1(4.5 \%)$ & $8(36.4 \%)$ & $4(16.7 \%)$ & $8(33.3 \%)$ \\
\hline 7-day death & 0 & $1(4.5 \%)$ & 0 & 0 \\
\hline 3-month death & 0 & $3(13.6 \%)$ & $1(4.2 \%)$ & $2(8.3 \%)$ \\
\hline 1-year death & 0 & $1(4.5 \%)$ & 0 & $5(20.8 \%)$ \\
\hline 3-year death & $1(4.5 \%)$ & $1(4.5 \%)$ & $3(12.5 \%)$ & 0 \\
\hline Survival time (days)* & 426 & 264 & 402 & 277 \\
\hline
\end{tabular}

* Median, range.

treatment group, and $43 \%, 29 \%$, and $29 \%$ in the untreated group. A significant difference in the survival rate was found between the combination treatment group and the other two groups (Figure 3a).

The results of Cox analysis are shown in Table 2. Based on 500 bootstrap samples, the univariate Cox regression analysis showed that concurrent $\operatorname{HCC}(P=$ 0.004), Child-Pugh Class $(P=0.002)$, severity of IAPFs $(P=0.040)$, portal vein embolus $(P=0.090)$, treatment selection (single treatment vs. untreated, $P=0.623$; combination treatment vs. untreated, $P=0.004)$, and early diagnosis of IAPFs $(P=0.014)$ showed a trend toward survival. In the multivariate analysis, treatment selection between the combination treatment and untreated groups was the only parameter significantly associated with survival $(P=0.002)$.

For the endpoint overall survival, patients with IAPF were divided into two groups based on the severity of their fistula. The cumulative survival rate at 1,2 , and 3 years was $86 \%, 56 \%$, and $56 \%$ in the mild-IAPF group $(n$ $=25)$ and $50 \%, 47 \%$, and $42 \%$ in the severe-IAPF group $(n=32)(p=0.054$, Figure $3 b)$. For IAPF-patients with or without $\mathrm{HCC}$, the overall survival showed significance $(p$ $=0.002$, Figure $3 \mathrm{c}$ ).

\section{Rebleeding}

The present study aimed at patients' occurrence of rebleeding after receiving interventions. The freeof-rebleeding period was defined as the time interval between the date of first treatment and the first episode of rebleeding after treatment or follow-up to 3 years. Patients concomitant $\mathrm{HCC}$ had the higher risk of rebleeding after the interventions ( $p=0.0016$, Figure $3 d)$. Nevertheless, the cumulative probability of remaining free of rebleeding in 3 years revealed no significant difference between the two interventional groups $(P=0.62$, Figure $3 \mathrm{e})$. The non-rebleeding rate at 6 months, and 1 and 2 years was $71 \%, 56 \%$, and $43 \%$ in the combination treatment group and $50 \%, 41 \%$, and $26 \%$ in the single treatment group, respectively.

Stratification analysis was performed on patients in the severe-IAPF group. The trend that combination treatment $(n=5)$ was superior to single treatment $(n=20)$ is shown in Figure $3 \mathrm{f}$.

Further analysis was conducted on the patients receiving interventions, who were divided into two groups according to the first procedure they underwent. Twenty-two of them first received endoscopic treatment, and the other 24 patients first underwent TAE. A total of 26 patients developed rebleeding. Twelve (54.5\%) in the group first received endoscopic treatment, and 14 (56.0\%) first received TAE procedures. Time to rebleeding and outcomes are listed in Table 3.

\section{DISCUSSION}

In this study conducted in a tertiary hospital famous for liver disease, the CT reports from more than 10,000 patients with liver disease and 451 patients diagnosed with IAPFs were screened. About half of the patients with IAPF had gastroesophageal varices with a $12.9 \%$ bleeding rate. The results showed that the combination of endoscopic treatment and TAE is the most optimal therapy.

Since the first IAPF case reported in 1892 [9], etiologies include trauma, iatrogenic complications of diagnostic and therapeutic procedures[10], congenital lesions, arteriovenous shunting within HCC, spontaneous development of a fistula, and so on [1]. In the present study, the major etiology of IAPFs is HCC, that existed in $61.4 \%$ of patients. In line with previous studies on IAPFs, it was found that ascites were the common manifestations 
a

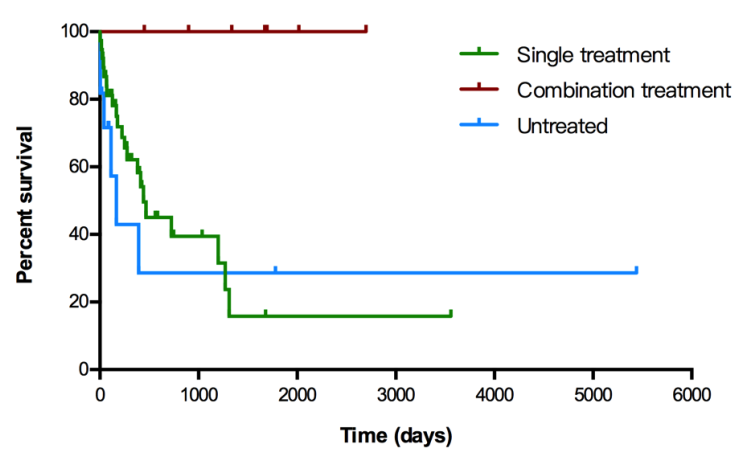

C

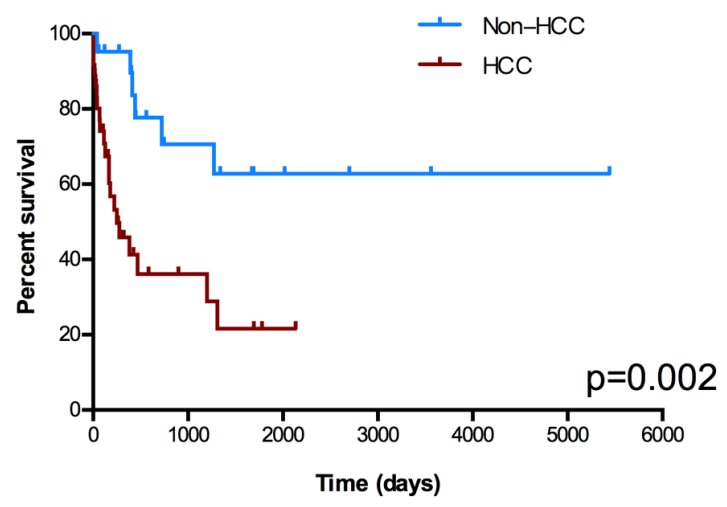

e

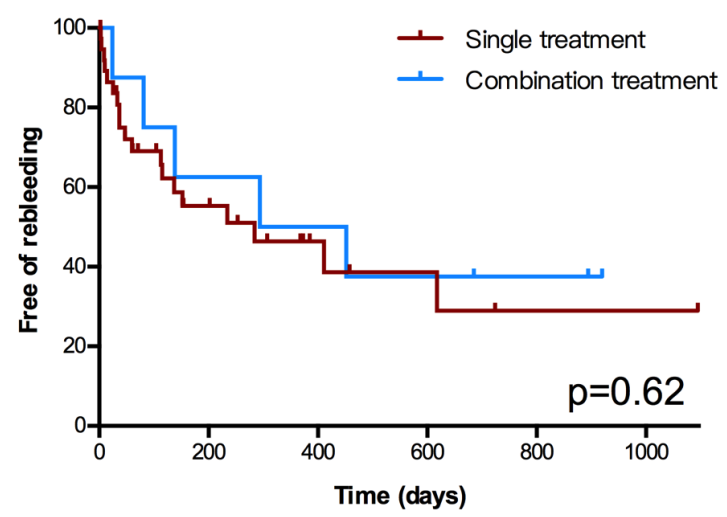

b

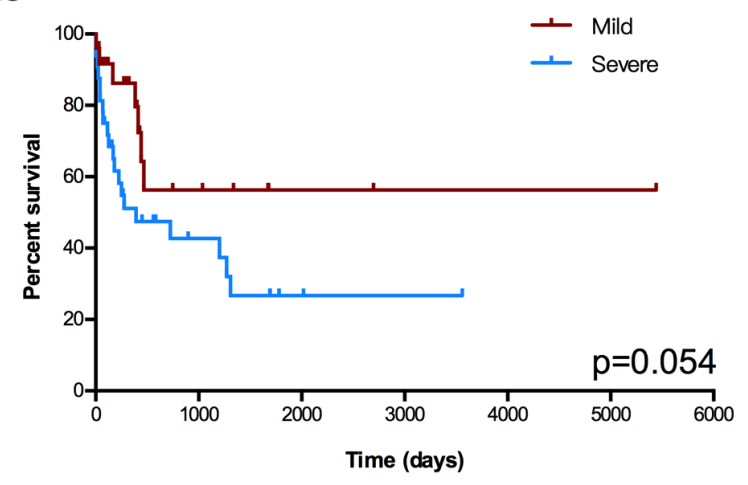

d

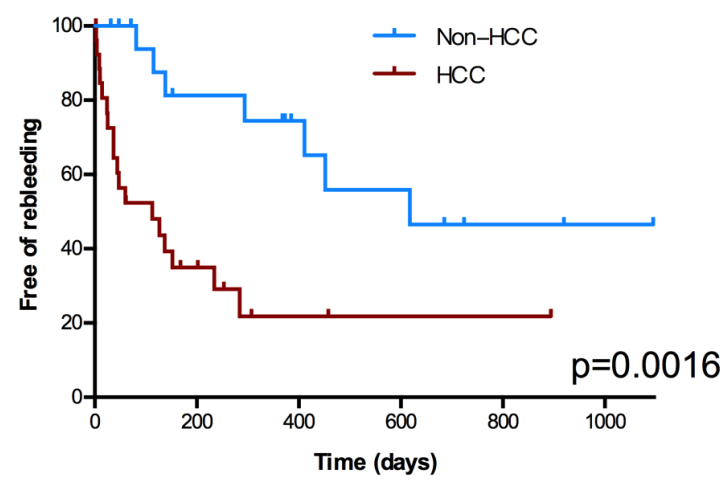

$\mathbf{f}$

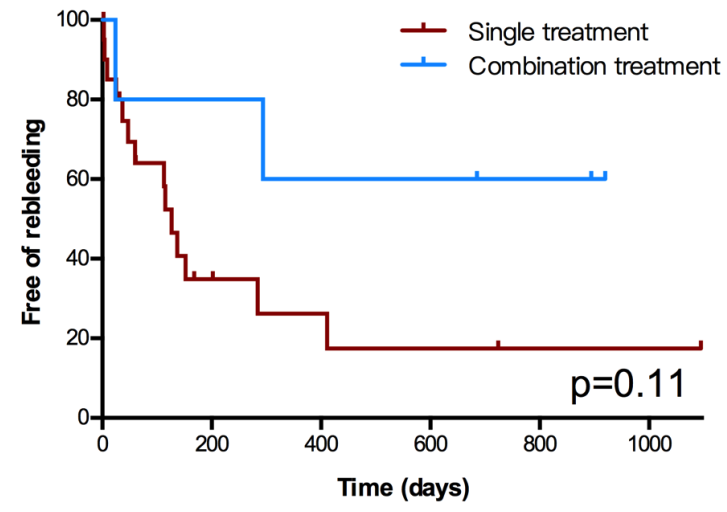

Figure 3: Kaplan-Meier estimates of overall survival and rebleeding. a. Overall survival stratified by the three groups. b. Overall survival stratified by the severity of IAPFs. c. Overall survival stratified by patients with or without Hepatocellular Carcinoma (HCC). d. Three-year rebleeding rate stratified by with or without HCC in patients received treatment $\mathbf{e}$. Three-year rebleeding rate in the two interventional groups in all patients. f. Three-year rebleeding rate among patients with severe IAPF in the two interventional groups. 
of gastrointestinal bleeding while hepatic encephalopathy was less common [1]. Also, 46 patients in the present study had ascites while 26 patients presented with massive ascites.

The importance of secondary prophylaxis after variceal bleeding in patients with IAPF is considerably acknowledged, yet the therapeutic approaches are limited. Currently, no therapeutic strategy exists regarding the management of varices in patients with IAPF via endoscopy, and the predominant treatment in these patients is TAE. However, patients suffered refractory rebleeding after TAE procedures are common. Previous studies showed that long-standing IAPFs might be associated with structural liver changes, including vascular changes, minimal peripheral fibrosis, and conserved lobular architecture [11]. Radiologic interventions can only deal with the fistula-feeding vessel(s), but the sustained presence of gastroesophageal varices remains a high risk of variceal hemorrhage. Especially in patients with mild severity of IAPF, TAE can only reduce a small amount of portal pressure; the remaining varices have the high risk of rebleeding.

Combination treatment has the best efficacy especially in patients with severe IAPF, while single treatment using either endoscopic treatment or TAE is only slightly better than no treatment. The reasons may be that TAE simply embolizes the fistula and partially reduces the portal pressure added by the shunt. The original cirrhotic portal hypertension is still present and coexists with the gastroesophageal varices. Thus, the risk of rebleeding and death is not reduced. As for endoscopic treatment, however, it has the unsatisfactory outcomes in patients with HVPG larger than $20 \mathrm{mmHg}$ [12]. For these patients with IAPF, the existing arterial-venous shunt increases the portal pressure compared with the general cirrhotic patients. Hence, the efficacy of endoscopic treatment for variceal bleeding is not achieved. The symptoms of IAPFs are dependent on the severity of fistula, and the amount of blood shunted that increases portal pressure. Single endoscopic or TAE procedures have equivalent efficacy in IAPFs, which corresponds to their own efficacy. The combination of endoscopic and TAE treatment achieves the optimal efficacy by decreasing portal pressure through embolization of the fistula and alleviating the rebleeding risk by endoscopic treatment.

Remarkably, seven patients received splenectomy before the diagnosis of IAPF and 47 patients $(82.5 \%)$ diagnosed with IAPF after the first variceal bleeding. It indicated that IAPFs were easy to be misdiagnosed in clinical practice. In Cox regression analysis, treatment selection between untreated and combination treatment groups is the only parameter that influences survival. It is essential to achieve a better prognosis by prompt CT angiography when variceal bleeding occurs and have the optimal treatment selection. The early detection of the existence of IAPF helps in thoughtfully considering these patients and providing them essential treatment.
The free-of-rebleeding period was similar in both the combination and single-treatment groups. That is, either TAE or endoscopic treatment could not prevent variceal bleeding. Further stratification analysis showed that no matter what the first receiving procedure was, the rebleeding rate and overall survival had no difference.

Endoscopic procedures, including esophageal ligation, sclerotherapy, and tissue adhesive injection, were widely recommended in managing gastroesophageal hemorrhage [6][13]. In patients with mild-severity IAPF, the outcome of endoscopic treatment was slightly better than that of TAE procedures because these patients were similar to other patients with cirrhotic variceal bleeding. However, no recommendation for the management of variceal hemorrhage in patients with IAPF was found. Liver transplantation is considered the final method of rescuing patients with unresectable collateralization [14]. Four patients in the present study underwent liver transplantation for their primary liver disease, and they were alive without further complications during followup. In our experience, most of the IAPFs could be well managed by radiological intervention and endoscopic procedures.

The present study had some limitations. First, in this retrospective study, different sample size among three groups and differences in the severity of IAPFs and disease might have influenced the results when comparing groups. With the bootstrap sampling Cox regression model, combination treatment appeared to be the best therapeutic strategy. Second, the study included patients with IAPF regardless of any clinical status; consequently, some of the patients in poor condition introduced bias into the overall survival.

Although IAPF is uncommon, it should be considered as one of the differential diagnosis when refractory variceal bleeding occurs. For those diagnosed with IAPF, a combination of endoscopic management and radiologic interventional procedures is the most optimal treatment to avoid secondary massive UGIB and improve prognosis, especially in patient with severe IAPF.

\section{Abbreviations}

IAPF: Intrahepatic arterio-portal fistula; UGIB: Upper gastrointestinal bleeding; TAE: Transcatheter arterial embolization; CT: Computed tomography; HCC: Hepatocellular carcinoma.

\section{Authors contributions}

Prof. Chen Shiyao proposed the study. Dr. Huang Xiaoquan performed the research, acquisition and analysis of data, and drafted the article. Prof. Chen Shiyao, Dr. Wang Jian and Dr. Li Feng performed the endoscopic treatments. Dr. Zhang Wen and Dr. Luo Jianjun performed 
the radiological interventions. Dr. Sheng Ruofan and Dr. $\mathrm{Xu}$ Pengju made the imaging diagnosis. Dr. Zhang Wen and Dr. Liu Chengfeng analysis and interpretation of the study.

\section{ACKNOWLEDGMENTS}

We would like to thank all the staff in Department of Gastroenterology, Endoscopy Center and Endoscopy Research Institute, Liver Cancer Institute, Department of Interventional Radiology and Department of Radiology, Zhongshan Hospital, Fudan University for assistance. All authors declare no conflicts of interest that are relevant to the manuscript.

\section{CONFLICTS OF INTEREST}

The authors declared that they had no conflicts of interest with respect to their authorship or the publication of this article.

\section{FUNDING}

The study was supported by Innovation Fund of Science and Technology Commission of Shanghai Municipality (No. 15411950500) and partly supported by the Fund of National Science \& Technology Pillar Program (No. 2013BAI09B14). The funders had no role in the study design, data collection and analysis, decision to publish, or preparation of the manuscript.

\section{REFERENCES}

1. Vauthey JN, Tomczak RJ, Helmberger T, Gertsch P, Forsmark C, Caridi J, Reed A, Langham MR, Lauwers GY, Goffette P, Lerut J. The arterioportal fistula syndrome: clinicopathologic features, diagnosis, and therapy. Gastroenterology. 1997; 113: 1390-401.

2. Tannuri ACA, Tannuri U, Lima FR, Ricardi LRS, Leal AJG, da Silva MM. Congenital intrahepatic arterioportal fistula presenting as severe undernutrition and chronic watery diarrhea in a 2-year-old girl. J Pediatr Surg. 2009; 44: e19-22. doi: 10.1016/j.jpedsurg.2009.07.027.

3. Takagi K, Yagi T, Yoshida R, Shinoura S, Umeda Y, Nobuoka D, Watanabe N, Kuise T, Sui K, Hirose A, Tsuboi M, Ogasawara M, Iwasaki S, et al. A successful case of deceased donor liver transplantation for a patient with intrahepatic arterioportal fistula. Hepatol Res. 2016; 46:1409-1415. doi: 10.1111/hepr.12701.
4. Lumsden AB, Allen RC, Sreeram S, Atta H, Salam A. Hepatic arterioportal fistula. Am Surg. 1993; 59: 722-6.

5. Iwaki T, Miyatani H, Yoshida Y, Matsuura K, Suminaga Y. Gastric variceal bleeding caused by an intrahepatic arterioportal fistula that formed after liver biopsy: a case report and review of the literature. Clin J Gastroenterol. 2012; 5: 101-7. doi: 10.1007/s12328-011-0277-y.

6. Tripathi D, Stanley AJ, Hayes PC, Patch D, Millson C, Mehrzad H, Austin A, Ferguson JW, Olliff SP, Hudson M, Christie JM, Lowsley-Williams B, Guo K. UK guidelines on the management of variceal haemorrhage in cirrhotic patients. Gut. 2015. doi: 10.1136/gutjnl-2015-309262.

7. Guzman EA, McCahill LE, Rogers FB. Arterioportal fistulas: introduction of a novel classification with therapeutic implications. J Gastrointest Surg. 2006; 10: 543-50. doi: 10.1016/j.gassur.2005.06.022.

8. Choi BI, Lee KH, Han JK, Lee JM. Hepatic arterioportal shunts: dynamic CT and MR features. Korean J Radiol. 2002; 3: 1-15. doi: 10.3348/kjr.2002.3.1.1.

9. Sachs R. Zur casuisitik der gefasserkrankungen. [Article in German]. Dtsch Med Wochenschr. 1892; 18: 443-7.

10. Bedell JE, Keller FS, Rösch J. Iatrogenic intrahepatic arterial-portal fistula. Radiology. 1984; 151: 79-80. doi: 10.1148/radiology.151.1.6701341.

11. Zuidema GD, Gaisford WD, Abell MR, Brody TM, Neill SA, Child CG. Segmental portal arterialization of canine liver. Surgery. 1963; 53: 689-98. doi: 10.5555/ uri:pii:0039606063900242.

12. Garcia-Tsao G, Abraldes JG, Berzigotti A, Bosch J. Portal hypertensive bleeding in cirrhosis: Risk stratification, diagnosis, and management: 2016 practice guidance by the American Association for the study of liver diseases. Hepatol Baltim Md. 2017; 65: 310-35. doi: 10.1002/ hep. 28906.

13. de Franchis R, Faculty BV. Expanding consensus in portal hypertension: Report of the Baveno VI Consensus Workshop: Stratifying risk and individualizing care for portal hypertension. J Hepatol. 2015; 63: 743-52.

14. Duman JD, Johnson SP, Trotter JF. Arterioportal fistula requiring liver transplantation. Liver Transplant. 2006; 12: 1904-5. doi: 10.1002/lt.20989. 